\title{
Un rilievo speditivo di emergenza. Forte San José a Cartagena de Indias (Colombia)
}

An expeditious emergency survey. Fort San José in Cartagena de Indias (Colombia)

\author{
Alfonso Cabrera Cruza ${ }^{\text {a, }}$, Massimo Leserri ${ }^{\text {b }}$, Gabriele Rossi ${ }^{\text {c }}$, Ricardo Zabaleta ${ }^{\text {d }}$

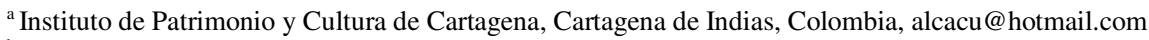 \\ ${ }^{\mathrm{b}}$ Universidad Pontificia Bolivariana, Monteria, Colombia, massimo.leserri@upb.edu.co \\ ${ }^{\text {c } D i p a r t i m e n t o ~ d i ~ S c i e n z a ~ d e l l ' I n g e g n e r i a ~ C i v i l e ~ e ~ d e l l ' A r c h i t e t t u r a ~-~ P o l i t e c n i c o ~ d i ~ B a r i, ~ B a r i, ~ I t a l y, ~ g a b r i e l e . r o s s i @ ~ p o l i b a . i t ~}$ \\ ${ }^{\mathrm{d}}$ Universidad de San Buenaventura, Cartagena de Indias, Colombia, rizapu@ gmail.com
}

\begin{abstract}
The recent methods of survey and modeling based on digital images or laser scanner technologies, today widely tested, are an exceptional support for the rapidity of acquisition, especially in emergency situations where it is necessary to quickly acquire a documentation in order not to stay long in a place.

The Fort San Jose, designed by Juan de Herrera between 1714 and 1725 and completed by Antonio de Arévalo after almost 50 years, completes the defense of navigable access to the bay of Cartagena de Indias between the island of Bocachica and Isla Bomba. Placed on an artificial island in front of the fort of $\mathrm{S}$. Ferdinando it has undergone frequent flooding in recent years due to a sinking process.

Studies relating to emergency expeditious surveys are limited and episodic, detailed in most cases in the archaeological field where urgent excavations must be carried out and phenomena and situations that can be lost must be recorded rapidly. The Italian Ministry of Heritage and Cultural Activities in 2013 prepared card systems to document the damage following natural disasters.

In the case of study, modern surveying methods are used, integrating the use of laser scanner technologies for accessible areas and aerial photogrammetry for those facing the sea. The objective is to draw up an architectural survey that documents the state of consistency of the fortress that has never been detected to date and in this way initiate a monitoring of the ongoing processes. At the same time, it constitutes an indispensable cognitive support for possible activities and intervention strategies that aim to put an end to instability.
\end{abstract}

Keywords: Architectural survey, emergency survey, Cartagena de Indias.

\section{Introduzione e Stato dell'Arte}

Forte San José, monumento nazionale con decreto 1911 del 2 novembre 1995 insieme all'intero sistema difensivo di Cartagena de Indias, sorge su un isolotto in parte artificiale sito all'imboccatura della baia di Cartagena di fronte a Forte San Ferdinando. Disegnato da Juan de Herrera tra il 1714 e il 1725 ed ultimato da Antonio de Arévalo dopo circa 50 anni, completa la difesa dell'accesso navigabile alla baia tra l'isola di Bocachica e Isla Bomba.
L'importanza commerciale del porto colombiano nel mare dei Caraibi e le crescenti esigenze del turismo croceristico hanno reso indispensabile dragare ripetutamente l'accesso alla baia soggetto ad insabbiamento per consentire il passaggio delle grandi navi porta containers e da crociera. Tali operazioni mettono a repentaglio la conservazione dei due forti ed in particolare quello di San José che sprofonda progressivamente. 


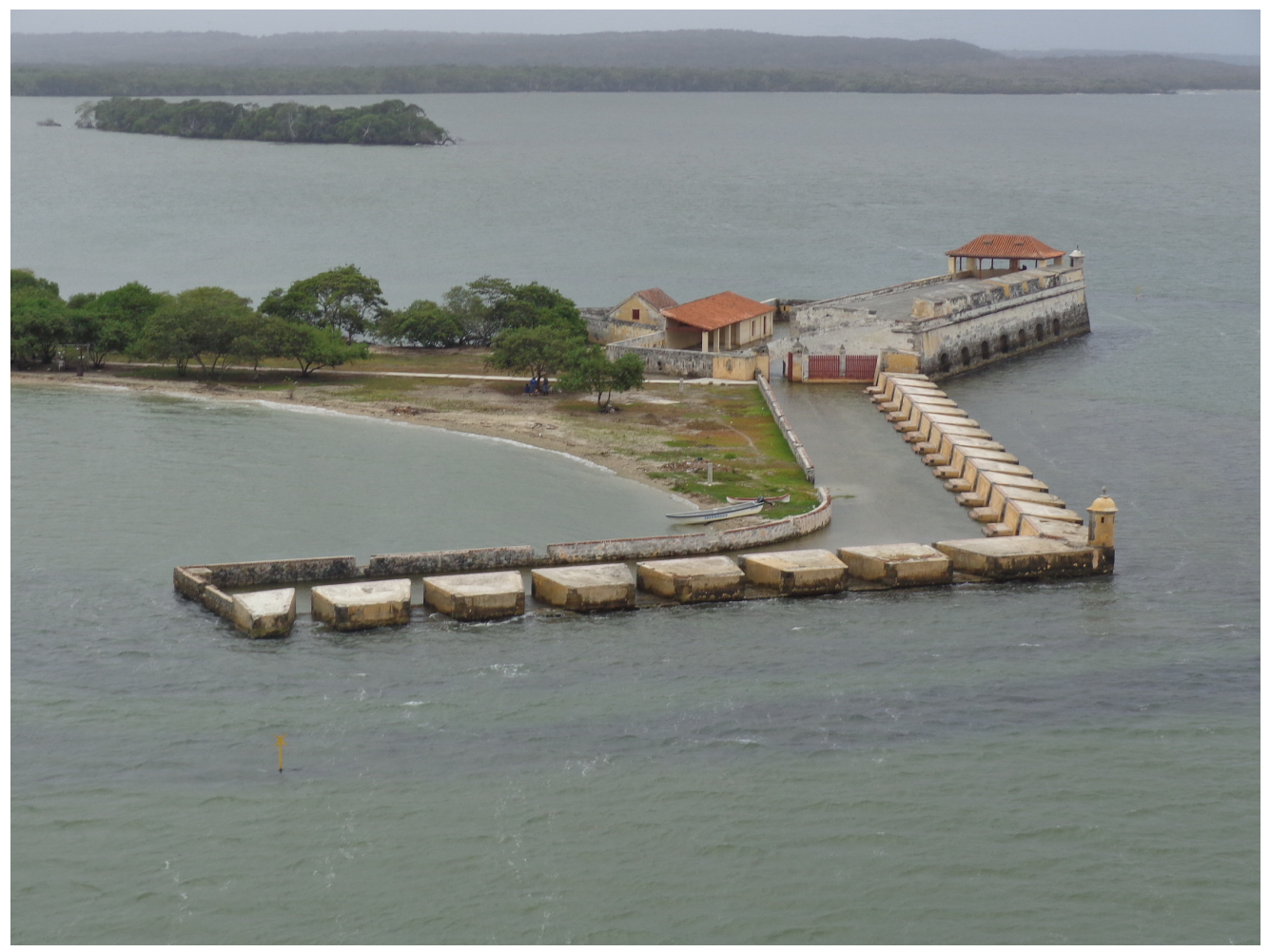

Fig. 1. Vista del Forte di San José.

Quest'ultimo sorge sull'estremità settentrionale dell'isolotto in parte artificiale all'ingresso meridionale della baia e si compone di un corpo originario con numerosi ambienti coperti e la polveriera ed un corpo più basso costituito da una batteria a forma di L rivolta verso il mare aperto ed in parte verso Forte San Ferdinando.

Le rappresentazioni conservate a Madrid presso il Servicio Historico Militar Plano de la Bateria S. Ioseph dela Isla situata ala entrada de Bocachica haziende frente al Castillo y Canal e Elevacion en Perspectiva Militar dela Bateria S. Ioseph risalenti al 1730 mostrano la consistenza del forte prima dell'ampliamento e l'aggiunta della nuova batteria.

A quell'epoca il forte presenta una forma irregolare con le postazioni per i cannoni sul lato d'ingresso alla baia, su una escollera de Pietra probabilmente di origine artificiale; all'interno con accesso da due porte -Puerta principal sul lato settentrionale e Puerta que sale al Manglar sul lato sud-orientale- si sviluppano una serie di ambienti coperti tra cui una cisterna e una cap- pella intitolata a Santa Barbara; lungo il perimetro della fortificazione si dispongono cinque garitte di cui tre in corrispondenza della batteria, una sulla porta d'ingresso sud-orientale ed una sul vertice orientale delle mura.

Una ricca e consolidata letteratura attesta l'interesse per le fortificazioni del Caribe (Angulo Íñiguez, Sánchez Catón, 1942; Zapatero, 1978) e per quelle di Cartagena in particolare; ci si riferisce agli studi degli anni '70 ad opera di Zapatero (1969, 1978), quelli successivi opera invece di Segovia (1982) sino a quelli più attuali di Cabrera Cruz (2018).

Il recente studio condotto su Forte San Ferdinando, sebbene non prenda avvio da un rilievo sistematico e rigoroso del forte e si basi solo sulla documentazione esistente e su una attenta analisi della tecnica costruttiva, costituisce una lettura indispensabile per comprendere le dinamiche e le cause alla base dei numerosi problemi riscontrati e che accomunano i due forti che si fronteggiano (Galassi, Paradiso, Benedetti, 2013, pp. 136-158). 


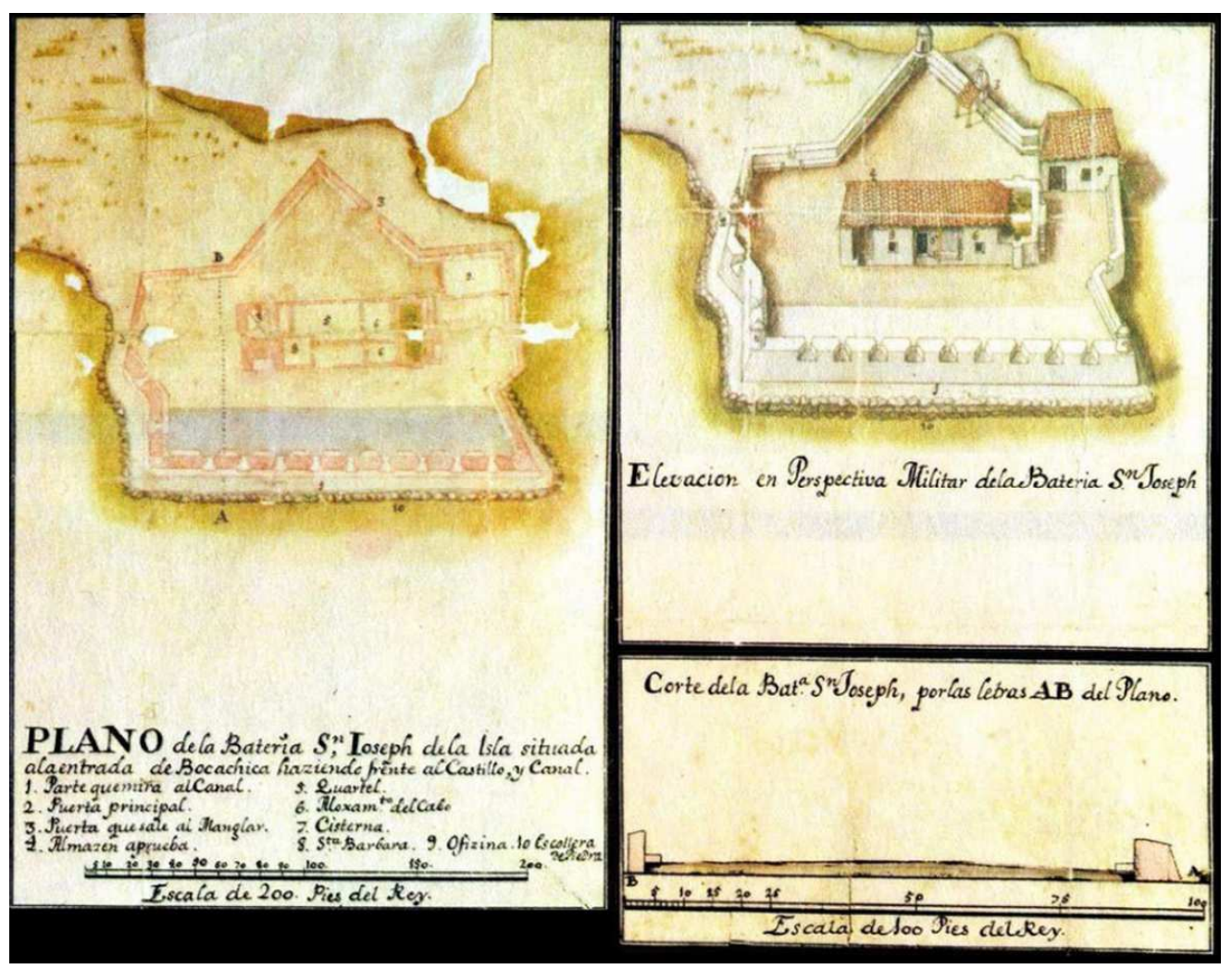

Fig. 2. Planimetria, sezione e vista prospettica della Battería di San José, di Juan de Herrera y Sotomayor, 1730. Documentazione custodita presso il Servicio Histórico Militar, Madrid.

Limitati ed episodici invece sono gli studi in merito alla esperienza di rilievo condotte in situazioni di emergenza, sebbene in ambito archeologico sia possibile trovare numerosi contributi che suggeriscono strumenti e metodi da utilizzare in situazioni dove l'incalzare degli eventi e la carenza cronica di mezzi e risorse rende necessario intervenire in maniera speditiva (Angelini, Colosi, Fentress, Filippone, Gabrielli, 2007, pp. 141-158). A seguito di calamita naturali invece alcune pubbliche amministrazioni hanno sviluppato protocolli e strategie per registrare rapidamente e rilevare in maniera sintetica gli effetti degli eventi nefasti per un controllo immediato dei danni e per valutare l'agibilità stessa degli immobili.

A tale scopo il ricorso a metodi e strumenti di grande semplicità, la conoscenza delle regole base della geometria proiettiva e l'utilizzo di reticoli quadrangolari o triangolari in ambito archeologico consentono un'attività di documentazione sempre valida a sopperire le esigenze che l'emergenza di volta in volta propone (Malesani, Valdambrini, 2000; Marino, 2015).

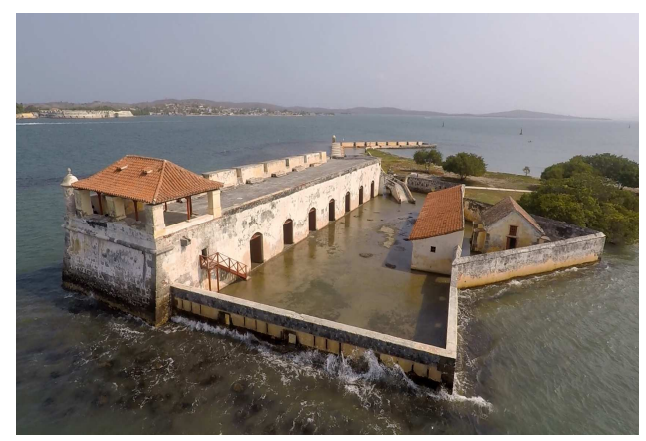

Fig. 3. Vista di Forte San José con l'alta marea.

\section{Metodologia}

Le tecnologie laser scanner invece, oramai consolidate da anni, e le più recenti tecniche di structure from motion permettono grazie ai nuovi algoritmi la creazione di modelli $3 \mathrm{~d}$ a partire 
da opportune sequenze di riprese fotografiche. I modelli tridimensionali generati e le restituzioni ortografiche che si ottengono contribuiscono a modificando in maniera progressiva il modo di vedere e rappresentare il mondo reale (Fassi, Campanella, 2017, pp. 313-319); i software sviluppati in questo ambito consentono di raggiungere un livello di dettaglio e precisione sempre maggiore calibrando di volta in volta i parametri idonei (Alidoost, Arefi 2017, pp. 55-61; Fallavolita, Ballabeni, Foschi, R.; Perugini, 2015, pp. 31-40).

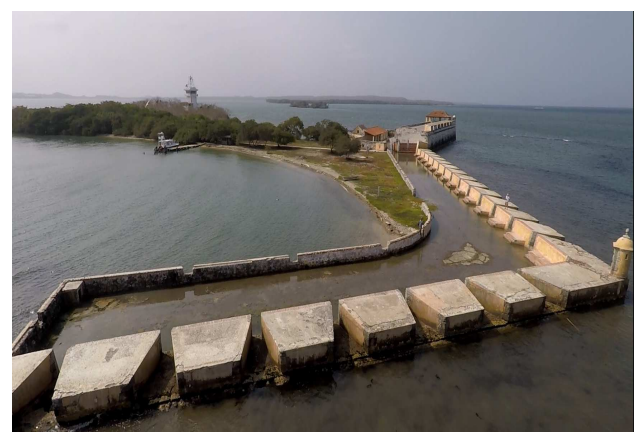

Fig. 4. Vista di Forte San José con l'alta marea.

L'attività speditiva proposta nasce dunque dall'esigenza di documentare la situazione del forte che sprofonda a causa delle attività di drenaggio condotte ripetutamente per consentire l'accesso delle grandi navi alla baia d Cartagena.

Il Ministero della Cultura colombiano -presa consapevolezza dei danni- predispone già nel 2005 con risoluzione n. 97 del 28 dicembre una barriera di protezione permanente per ridurre l'impatto delle onde sulla antica struttura. Lo stesso ministero poi preoccupato per la conservazione del forte promuove uno Estudios técnicos y proyecto de restauracio integral del fuerte de San Ferdinandoy el fuerte-bateria San José en Bocachica - Cartagena, Bolivar coordinato dall'arch. José Alberto Herrera Diaz che tra le numerose attività include anche rilievi topografici ed architettonici del forte.

I rilievi allora condotti in ambito di tali studi ministeriali sembra siano in fase di revisione ed approvazione e non sono ancora consultabili; pertanto ad oggi non si ha ancora un quadro esatto della consistenza architettonica del forte.

Ci si trova così, a causa di eventi improvvisi ed inaspettati, a dover intervenire per documentare lo stato di consistenza di manufatti architettonici compromessi e a rischio di perdita. Le calamità naturali di natura diversa che in maniera sempre più frequente si abbattono sul nostro pianeta costituiscono la principale emergenza e sono quindi la motivazione per le attività speditive di rilievo al fine di documentarne i danni e valutare lo stato di consistenza degli immobili compromessi. Agli eventi naturali si aggiungono poi gli eventi bellici con la perdita o compromettendo la conservazione di numerosi monumenti patrimonio dell'umanità.

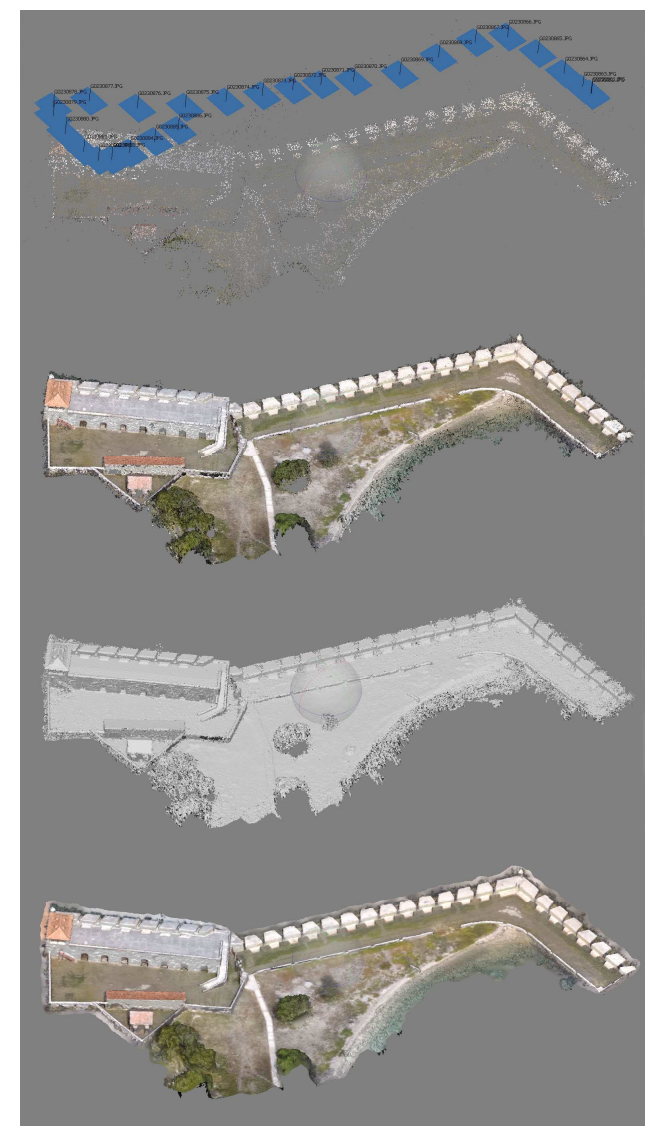

Fig. 5. Elaborazioni con Metashape di Agisoft.

Oltre a situazioni di emergenza determinate da calamità naturali esistono poi situazioni, come in questo caso, determinate dalla normale attività dell'uomo; il rinvenimento, nella maggior parte dei casi di resti archeologici imprevisti ed inaspettati, rende necessario un'attività di rilievo e documentazione da condursi in tempi brevi per non ostacolare il proseguimento delle attività stesse. 


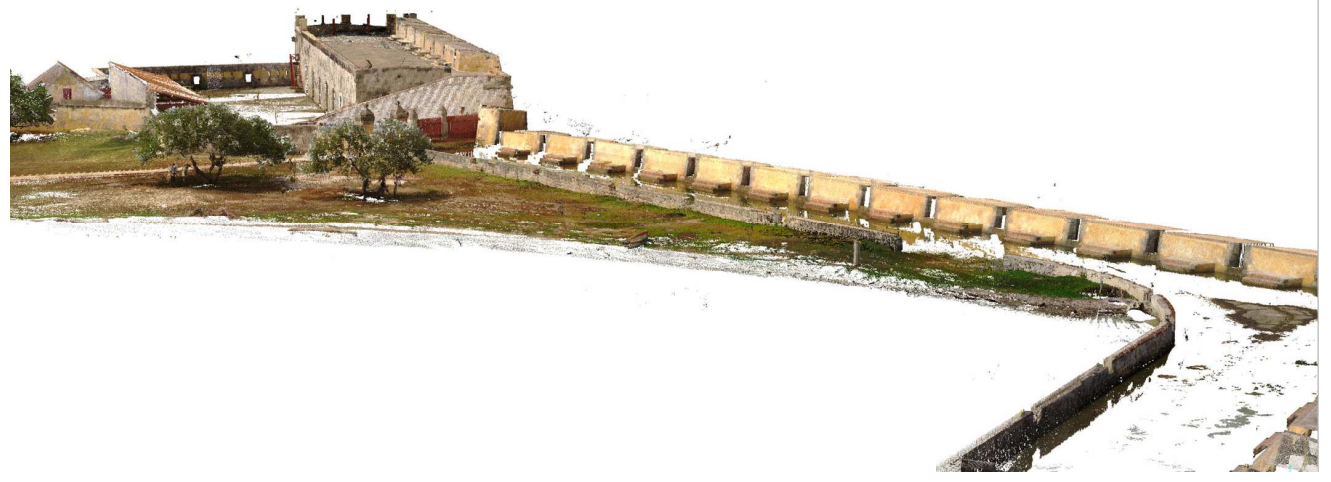

Fig. 6. Vista della nuvola di punti.

Nel nostro caso le opere di drenaggio condotte e le ripercussioni sul manufatto hanno reso necessaria un'attività speditiva nel febbraio 2019 con l'obiettivo di redigere un rilievo architettonico per documentare lo stato di consistenza della fortezza ad oggi, avviare un monitoraggio dei processi in atto ed allo stesso tempo costituire un supporto conoscitivo indispensabile per possibili attività e strategie di intervento che mirino a porre fine ai dissesti.

L'attività speditiva di cui si presentano i primi risultati è promossa dal IPCC Instituto de Patrimonio y Cultura de Cartagena de Indias, istituzione distrettuale pubblica che ha tra gli obiettivi la promozione, la programmazione, il coordinamento ed il finanziamento delle attività culturali ma anche la gestione, documentazione e protezione del patrimonio architettonico e tangibile della città di Cartagena.

In occasione del sopralluogo di fine febbraio come mostrano le riprese- l'intero piano di campagna risulta sotto il livello del mare e pertanto le attività di rilievo sono condotte in acqua. Infatti nelle prime ore del giorno il livello dell'acqua copre completamente il piano di campagna e supera in molti punti i $30 \mathrm{~cm}$.; progressivamente da fine mattinata al primo pomeriggio il livello dell'acqua scende con la bassa marea ed emerge il piano di campagna cosparso dai resti delle maree.

L'attività speditiva integra il rilevamento laser scanner con le tecniche di Structure from motion realizzate con riprese aeree da drone e terrestri da imbarcazione ed in un'unica giornata sono acquisite 27 nuvole di punti con un laser scanner della ditta Faro modello Focus M70 assicurando la copertura degli ambienti accessibili e di tutti gli spazi aperti raggiungibili. Contemporaneamente le riprese fotografiche da drone documentano le coperture e le parti inaccessibili da terra, riprese terresti da imbarcazione invece quelle accessibili solo via mare.

Il drone utilizzato è della GoPro Karma sul quale è istallata una camera HERO6 che ha consentito di acquisire immagini con risoluzione di $12 \mathrm{MP}$; la camera per le prese terrestri è invece una $\mathrm{Ni}$ kon modello D5300 con riprese sempre da 12 MP.

Le nuvole di punti sono elaborate e registrate con il software Scene 7.0 della stessa Faro generando un'unica nuvola di punti dell'intera struttura fortificata che si sta procedendo ad integrare i dati ricavati dalle riprese fotografiche aeree e terrestri elaborati con il software Metashape di Agisoft.

Sebbene le operazioni di restituzione dei dati non siano ancora concluse, le prime elaborazioni mostrano la ricchezza delle informazioni sufficienti a redigere una prima documentazione dello stato di consistenza ed avviare un'attività di monitoraggio e salvaguardia del forte.

\section{Conclusioni}

L'esperienza condotta qui si inserisce in un filone di attività di rilievo speditivi d'emergenza che trova nelle nuove tecnologie e tecniche supporti rapidi ed efficaci indispensabili a rendere agevoli le operazioni di documentazione lì dove non c'è il tempo per procedere in maniera tradizionale (Nannei, Fassi, Mirabelli Roberti, 2019). 


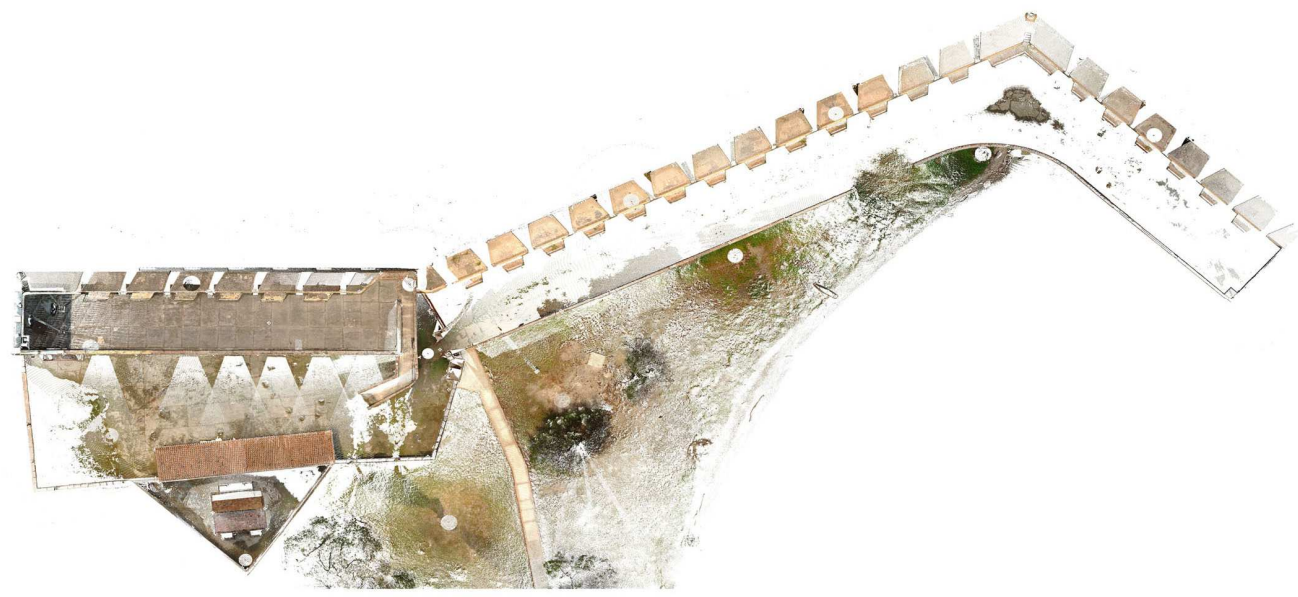

Fig. 5. Ortofoto da nuvola di punti.

La rapidità di acquisizione e la facilità di estrazione di immagini ortografiche a discapito di rappresentazioni d'architettura comporta però il rischio che le immagini elaborate costituiscano solo un momento della restituzione e non ne consentano l'interpretazione.

\section{Bibliography}

Alidoost, F.; Arefi, H. (2017). "Comparison of UAS-based photogrammetry software for 3D point clouds generation: a survey over a historical site", ISPRS Annals of the Photogrammetry, Remote Sensing and Spatial Information Sciences, vol. IV-4/W4, pp. 55-61, in https://doi.org/10.5194/isprs-annals-IV-4-W4-55-2017.

Angelini, A. ; Colosi, F.; Fentress, E.; Filippone, C.; Gabrielli, R. (2007). "Tecniche speditive per la ricostruzione tridimensionale dell'aerea archeologica di Villa Magna”, Archeologia e Calcolatori, 18, pp. 141-158.

Angulo Íñiguez, D.; Sánchez Catón, F.J. (1942). Bautista Antonelli. Las fortificaciones americanas del siglo XVI, Real Academia de la Historia Ed., Madrid.

Cabrera Cruz, A.R. (2018). El patrimonio arquitectónico y fortificaciones en Cartagena de Indias: Identidad, significado cultural y prospectiva, Tesis Doctorales, Universidad de Granada, Granada.

Fallavolita, F.; Ballabeni, M.; Foschi, R.; Perugini, G. (2015). "Semantic description of three dimensional models of Bologna porches", SCIRES-iT, 5, pp. 31-40.

Fassi, F.; Campanella, C. (2017). "From daguerrotypes to digital automatic photogrammetry. Applications and limits for the built heritage project", International Archives of the Photogrammetry, Remote Sensing and Spatial Information Sciences, 42, pp. 313-319.

Galassi, S.; Paradiso, M.; Benedetti, S. (2013). "Una contribución para conocer el Fuerte San Fernando de Bocachica, Cartagena de Indias, Colombia”, Revista M, 10, 2, pp. 136-158.

Marino L. (2015). Il rilievo in condizioni di emergenza. L'uso di reticoli, Altralinea Ed.

Malesani, F.; Valdambrini M. (2000). "Il rilievo di emergenza", in Nenci, C., ed., Restauro archeologico, Didattica e ricerca 1997-1999, Alinea Editrici, Firenze, pp. 49-56.

Nannei, V.M.; Fassi, F.; Mirabelli Roberti, G. (2019). "Photogrammetry for quick survey in emergency conditions: the case of Villa Galvagnina", International Archives of the Photogrammetry, Remote Sensing and Spatial Information Sciences, 42, pp. 835-842

Segovia Salas, R. (1982). Las fortificaciones de Cartagena de Indias. Estrategia y Historia, Carlos Valencia Editores Bogotá Ed., Cartagena.

Zapatero, J.M. (1969). Las fortificaciones de Cartagena de Indias. Estudio asesor para su restauración, Talleres Gráficos de la Vda. de C. Bermejo Ed., Madrid.

Zapatero, J.M. (1978). Las fortificaciones Abaluardata en America, Instituto de Cultura Puertorriquena, San Juan de Porto Rico, Barcelona.

Zapatero, J.M. (1979). Historia de las fortificaciones de Cartagena de Indias, Ediciones Cultura Hispánica Ed., Madrid. 